\title{
COEFFICIENT DIFFERENCES AND HANKEL DETERMINANTS OF AREALLY MEAN $p$-VALENT FUNCTIONS
}

\author{
JAMES W. NOONAN
}

ABSTRACT. With $p>0$, denote by $S_{p}$ the class of functions analytic and areally mean $p$-valent in the open unit disc. If $f \in S_{p}$, it is well known that $a(f)=\lim _{r \rightarrow 1}(1-r)^{2 p} M(r, f)$ exists and is finite. If $q \geq 1$ is an integer, denote the $q$ th Hankel determinant of $f$ by $H_{q}(n, f)$. In this paper the a symptotic behavior of $H_{q}(n, f)$, as $n \rightarrow \infty$, is related to $a(f)$. A typical result is: if $a(f)>0$, and if $p>q-3 / 4$, then

$$
\left|H_{q}(n, f)\right| / n^{2 p q-q^{2}} \sim\left|Q_{q}(p)\right|(\alpha(f) / \Gamma(2 p))^{q},
$$

where $Q_{q}$ is a polynomial of degree at most $q^{2}-q$. In the course of the proof, asymptotic results are proved concerning certain coefficient differences, and in particular concerning $\left|a_{n}\right|-\left|a_{n-1}\right| \cdot$

1. Introduction. A function $f$ analytic in $\gamma=\{z:|z|<1\}$ is said to be areally mean $p$-valent in $\gamma[5]$ if for all $R>0$

$$
\iint_{E(R)}\left|f^{\prime}(z)\right|^{2} \rho d \rho d \theta \leq p \pi R^{2},
$$

where $z=\rho e^{i \theta}$ and $E(R)=\{z \in \gamma:|f(z)|<R\}$. Throughout this paper we assume that $f$ is given by $f(z)=\Sigma_{0}^{\infty} a_{n} z^{n}$, where $f$ is normalized so that $\max _{1 \leq j \leq[p]}\left|a_{j}\right|=1$. The class of normalized areally mean $p$-valent functions will be denoted by $S_{p}$.

The problem of estimating the rate of growth of combinations of coefficients of functions in $S_{p}$ is well known (see [6, problem 6.14']). In addition to the well-known result of W. K. Hayman [3] that $\left\|a_{n}|-| a_{n-1}\right\|=O(1)$ for $f \in S_{1}$, this problem has been studied in [1], [7], [8], [9], and [10], to men-

Received by the editors June 21, 1973.

AMS (MOS) subject classifications (1970). Primary 30A34.

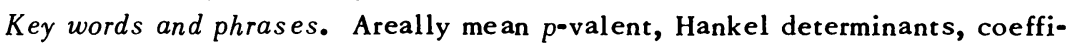
cient differences. 
tion only selected references. A closely related problem is the problem of estimating the rate of growth of the Hankel determinants for $f \in S_{p}$ (see [6, problem $6.13^{\prime}$ ]). If $q \geq 1$ is an integer, the $q$ th Hankel determinant of $f$ is defined to be

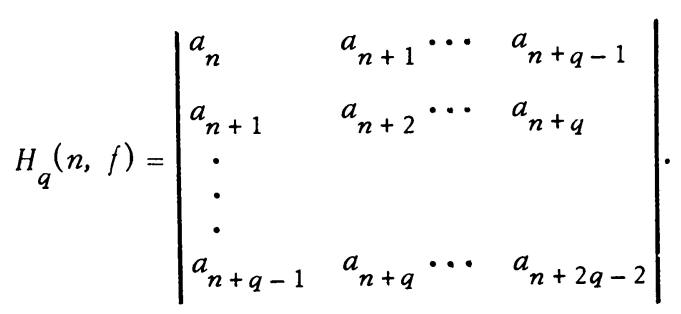

Ch. Pommerenke has shown [9] that for $p \geq 1, H_{q}(n, f)=O(1) n^{k \sqrt{ } q-q / 2}$, with $k=16 p \sqrt{ } p$. In particular, if $q$ is large relative to $p, H_{q}(n, f) \rightarrow 0$ as $n \rightarrow \infty$. For strictly univalent functions, Pommerenke has shown [10] that for $q \geq 2$, $H_{q}(n, f)=O(1) n^{3 / 2-(\beta+1 / 2) q}$, where $\beta>0$ is independent of $f$. In a recent paper with D. K. Thomas, the author showed [8] that $H_{q}(n, f)=O(1)_{n} 2 p q-q^{2}$ when $f \in S_{p}$ with $p \geq 2(q-1)$ and $q \geq 2$, and the exponent is best possible.

If $f \in S_{p}$, it is well known that $\alpha(f)=\lim _{r \rightarrow 1}(1-r)^{2 p} M(r, f)$ exists and is finite, where as usual $M(r, f)=\max |z|=r|f(z)|$ (see [1], [5]). It seems natural to expect that, at least when $p$ is relatively large, the value of $\alpha(f)$ should determine the asymptotic behavior of both coefficient combinations and Hankel determinants of $f \in S_{p}$. This conjecture is supported by the following results (see [2], [3]); (i) $H_{1}(n, f)=a_{n}$, and $\lim _{n \rightarrow \infty}\left|a_{n}\right| / n^{2 p-1}=$ $\alpha(f) / \Gamma(2 p)$ for $p>1 / 4$; (ii) if $\alpha(f)>0$, then $\left|a_{n}\right|-\left|a_{n-1}\right| \rightarrow \alpha(f)$ as $n \rightarrow \infty$ when $f \in S_{1}$. On the other hand, however, it is known [3] that (ii) need not hold if $a(f)=0$, and Hayman has also shown [4] that for $f \in S_{1}, H_{2}(n, f)=$ $o(1) n^{1 / 2}$ and the exponent is best possible. In each case, $p=1$ is "small", and $\alpha(f)$ does not determine the asymptotic behavior. In this paper we shall determine those values of $p$ for which $\alpha(f)$ determines the asymptotic behavior of $H_{q}(n, f)$ and of certain natural coefficient combinations.

2. Statement of results. With $j$ a nonnegative integer and $x$ any complex number, put

$$
\Delta_{j}(n, x, f)=\sum_{k=0}^{j}(-x)^{k}\left(\begin{array}{l}
j \\
k
\end{array}\right) a_{n+k},
$$

so $\Delta_{0}(n, x, f)=a_{n}, \Delta_{1}(n, x, f)=a_{n}-x a_{n+1}, \Delta_{2}(n, x, f)=a_{n}-2 x a_{n+1}+$ $x^{2} a_{n+2}$, and so forth. It was shown in [8] that if $f \in S_{p}$ and $p \geq j \geq 1$, then 
there exists $\left\{e^{i \theta} n\right\}$ such that $\Delta_{j}\left(n, e^{i \theta} n, f\right)=O(1) n^{2 p-j-1}$, and the exponent is best possible.

Theorem 1. Let $j$ be a positive integer.

(i) If $p>j$ and $f \in S_{p}$ with $a(f)=0$, there exists $\left\{e^{i \theta} n\right\}$ depending only on $f$ such that $\Delta_{j}\left(n, e^{i \theta_{n}}, f\right)=o(1) n^{2 p-j-1}$ as $n \rightarrow \infty$.

(ii) If $p \leq j$, there exists $f \in S_{p}$ with $a(f)=0$ such that for any sequence $\left\{e^{i \theta_{n}}\right\}$,

$$
\limsup _{n \rightarrow \infty} \frac{\left|\Delta_{j}\left(n, e^{i \theta_{n}}, f\right)\right|}{n^{2 p-j-1}}>0 .
$$

Note that with $j=1$, it follows from Theorem 1 that $\left|a_{n}\right|-\left|a_{n-1}\right|=$ $o(1) n^{2 p-2}$ if $p>1$ and $\alpha(f)=0$, while if $0<p \leq 1$, the asymptotic behavior of $\left|a_{n}\right|-\left|a_{n-1}\right|$ is not determined by the fact that $\alpha(f)=0$. Thus Theorem 1 generalizes the above-mentioned results of Hayman on the difference $\left|a_{n}\right|-\left|a_{n-1}\right|$.

Theorem 2. Let $q \geq 1$ be an integer. If $f \in S_{p}$ and $a(f)=0$, then

$$
H_{q}(n, f)=o(1) \begin{cases}n^{2 p-1}, & q=1, p>1 / 4 \\ n^{2 p q-q^{2}}, & q \geq 2, p>2(q-1) .\end{cases}
$$

We next consider the case $\alpha(f)>0$. It is well known $([1],[5])$ that in this case there exists a unique $\beta$ such that $\alpha(f)=\lim _{r \rightarrow 1}(1-r)^{2 p}\left|f\left(r e^{i \beta}\right)\right|$. We shall employ this notation consistently throughout this paper, and we shall also use the following notations

$$
\begin{array}{ll}
f_{\beta}(z)=\left(1-z e^{-i \beta}\right)^{-2 p}, & r_{n}=n /(n+1), \\
\alpha_{n}(f)=\left(1-r_{n}\right)^{2 p} f\left(r_{n} e^{i \beta}\right), & f_{n}(z)=\alpha_{n}(f) f_{\beta}(z) .
\end{array}
$$

In our next theorem we determine those values of $p$ for which $\Delta_{j}\left(n, e^{i \beta}, f\right)$ and $\Delta_{j}\left(n, e^{i \beta}, f_{n}\right)$ are asymptotically equivalent.

Theorem 3. If $j \geq 0, p>(2 j+1) / 4$, and $f \in S_{p}$ with $a(f)>0$, then as $n \rightarrow \infty$,

$$
\left(\Delta_{j}\left(n, e^{i \beta}, f\right)-\Delta_{j}\left(n, e^{i \beta}, f_{n}\right)\right) / n^{2 p-j-1} \rightarrow 0 .
$$

The restriction $p>(2 j+1) / 4$ is best possible in the sense that given $\epsilon>0$, 
there exists $p \in((2 j+1) / 4-\epsilon,(2 j+1) / 4)$ and $F \in S_{p}$ with $a(F)>0$ such that for any sequence $\left\{e^{i \theta_{n}}\right\}$,

$$
\lim _{n} \sup _{\infty} \frac{\left|\Delta_{j}\left(n, e^{i \theta} n, F\right)\right|}{n^{2 p-j-1}}=+\infty
$$

In $[8]$ it was shown that with $f_{0}(z)=(1-z)^{-2 p}$,

$$
\lim _{n \rightarrow \infty} \frac{\left|\Delta_{j}(n, 1, f)\right|}{n^{2 p-j-1}}=\left|\prod_{k=1}^{j}(k-2 p)\right|,
$$

and so the exponent $2 p-j-1$ above is clearly best possible. It was also shown in [8] that for $q \geq 1$ and for all $p>0$,

$$
\lim _{n \rightarrow \infty} \frac{\left|H_{q}\left(n, f_{0}\right)\right|}{n^{2 p q-q^{2}}}=\frac{\left|Q_{q}(p)\right|}{\Gamma(2 p)^{q}}
$$

where $Q_{q}$ is a nonconstant polynomial of degree at most $q^{2}-q$. Using Theorem 3, we prove

Theorem 4. Let $q \geq 1$ and $p>q-3 / 4$. If $f \in S_{p}$ and $\alpha(f)=$ $\lim _{r \rightarrow 1}(1-r)^{2 p}\left|f\left(r e^{i \beta}\right)\right|>0$, then

$$
\lim _{n \rightarrow \infty} \frac{\left|H_{q}(n, f)\right|}{n^{2 p q-q^{2}}}=\left(\frac{\alpha(f)}{\Gamma(2 p)}\right)^{q}\left|Q_{q}(p)\right|
$$

where $Q_{q}$ is as above. The restriction $p>q-3 / 4$ is best possible in the sense that given $q \geq 2$ and $\epsilon>0$, there exists $p \in(q-3 / 4-\epsilon, q-3 / 4)$ and $F \in S_{p}$ with $a(F)>0$ such that

$$
\limsup _{n \rightarrow \infty} \frac{H_{q}(n, F)}{n^{2 p q-q^{2}}}=\infty .
$$

3. The case $a(f)=0$. The proof of Theorem 1 is essentially the same as the proof of Theorem 2 of [8], and hence will not be repeated. The proof in [8] was based on estimates for certain integrals in terms of powers of $M(r, f)$. The full strength of the hypothesis $p \geq j$ in [8] was used only in the proof of Lemma 7-a of [8], where an integral was estimated in terms of $M(r, f)^{2(p-j) / p}$. If we now assume $p>j$, the exponent is positive, and since $a(f)=0$ implies $M(r, f)=o(1)(1-r)^{-2 p}$, our estimate in [8] holds with $o(1)$ replacing $O(1)$. Careful analysis shows that with $p>j$, all $O(1)$ terms in 
[8] can be replaced by $o(1)$, and so Theorem 1 is proved. Statement (ii) of Theorem 1 follows immediately from equation (6.3) of [8]. Theorem 2 follows directly from Theorem 1 upon using the method of $[8, \mathrm{p} .519]$.

4. The case $\alpha(f)>0$. We use the major-minor arc technique of Hayman [5] (see also [1]). With $r_{n}, a_{n}(f), f_{n}(z)$ as before and with $x_{n}=r_{n} e^{i \beta}, z_{n}=$ $r_{n} e^{i \theta}$, we have

$$
\left|\Delta_{j}\left(n, x_{n}, z f^{\prime}\right)-\Delta_{j}\left(n, x_{n}, z f_{n}^{\prime}\right)\right|
$$

$$
\leq A(p, j)\left\{\int_{E_{1}}\left|z-x_{n}\right|^{j}\left|f^{\prime}(z)-f_{n}^{\prime}(z)\right| d \theta+\int_{E_{2}}\left|z-x_{n}\right|^{j}\left|f^{\prime}(z)-f_{n}^{\prime}(z)\right| d \theta\right\},
$$

where (with $C$ an arbitrary constant) $E_{1}=\left\{\theta: 0 \leq|\theta-\beta| \leq C\left(1-r_{n}\right)\right\}, E_{2}=$ $\left\{\theta: C\left(1-r_{n}\right) \leq|\theta-\beta| \leq \pi\right\}$. From [1, Lemma 15] it follows that $f^{\prime}(z)-f_{n}^{\prime}(z)$ $=o(1) f_{n}^{i}(z)=o(1) n^{2 p+1}$, where as $n \rightarrow \infty, o(1)$ is uniform for $\theta \in E_{1}$. Since $\left|z-x_{n}\right|=r_{n}\left|e^{i(\theta-\beta)}-1\right|=O(1)|\theta-\beta|=O(1)_{n}^{-1}$ for $\theta \in E_{1}$, we conclude that as $n \rightarrow \infty$,

$$
\int_{E_{1}}\left|z-x_{n}\right|^{j}\left|f^{\prime}(z)-f_{n}^{\prime}(z)\right| d \theta=o(1) n^{2 p-j} .
$$

(Although the $O(1)$ terms above depend on $C$, note that $o(1) \rightarrow 0$ as $n \rightarrow \infty$, for any fixed $C$, and so we get $o(1)$ in (3).)

In order to estimate the second integral in (2), choose $\eta>0$ such that $p>(2 j+1) / 4+\eta j$. This is possible since $p>(2 j+1) / 4$. Set $a=(4 j)^{-1}-$ $1 / 2+\eta, \quad b=2 j(1+a) / p=p^{-1}(1 / 2+j+2 j \eta)$. With $z=r_{n} e^{i \theta}$, we apply the Cauchy-Schwarz inequality and find that

$$
\begin{aligned}
\int_{E_{2}} & \left|z-x_{n}\right|^{j}\left|f^{\prime}(z)\right| d \theta \\
& \leq\left\{\int_{E_{2}}\left|z-x_{n}\right|^{2 j}|f(z)|^{b} d \theta\right\}^{1 / 2}\left\{\int_{E_{2}}\left|f^{\prime}(z)\right|^{2}|f(z)|^{-b} d \theta\right\}^{1 / 2} \\
& =I_{1}^{1 / 2} I_{2}^{1 / 2} \text { say. }
\end{aligned}
$$

With $\lambda=2-b$, we have that $I_{2} \leq \int_{0}^{2 \pi}\left|f^{\prime}(z)\right|^{2}|f(z)|^{\wedge-2} d \theta$. Our choice of $\eta$ guarantees that $0<\lambda \leq 2$, and so from [5, Lemma 3.1]

$$
I_{2} \leq A(p, \lambda, f) M\left(r_{n}, f\right)^{\lambda} /\left(1-r_{n}\right)=O(1) n^{2 p \lambda+1} .
$$

We now estimate $I_{1}$. Choose $\epsilon \epsilon(0,2 p)$ so small that $2 j \eta(2-\epsilon / p)-$ $(j+1 / 2) \epsilon / p>0$. By [5, Theorem 2.9] there exists $C>0$ and $r_{0}<1$ such that 
$\left|f\left(r e^{i \theta}\right)\right| \leq(1-r)^{-\epsilon}|\theta-\beta|^{\epsilon-2 p}$ for $r_{0} \leq r<1, \theta \in\{\theta: C(1-r) \leq|\theta-\beta| \leq \pi\}$. Using the fact that $\left|z-x_{n}\right|^{2 j}=O(1)|\theta-\beta|^{2 j}$ and noting that $2 p b-\epsilon b-2 j$ $>1$ by our choice of $\epsilon$, we find that

$$
I_{1} \leq O(1)\left(1-r_{n}\right)^{-b \epsilon} \int_{C\left(1-r_{n}\right)}^{\infty}|\theta-\beta|^{\epsilon b+2 j-2 p b} d \theta=O(1) \frac{n^{2 p b-2 j-1}}{C^{2 p b-\epsilon b-2 j-1}} .
$$

Since $2 p b-\epsilon b-2 j-1>0$, and since $C$ can be chosen as large as desired, it follows that given $\delta>0$ there exists $C(\delta)$ and $r_{0}(\delta)$ such that

$$
I_{1}<\delta n^{2 p b-2 j-1} \text {. }
$$

Combining (4), (5), (6), and noting that $b+\lambda=2$, we see that

$$
\int_{E_{2}}\left|z-x_{n}\right|^{j}\left|f^{\prime}(z)\right| d \theta<\delta n^{2 p-j}
$$

for $n$ sufficiently large. Clearly the same estimate holds for $f_{n}^{\prime}$. Since $\delta>0$ is arbitrary and since $o(1)$ in (3) approaches zero once $\delta$ (and hence $C(\delta))$ has been fixed, we conclude from (2), (3), and (6) that

$$
\left(\Delta_{j}\left(n, x_{n}, z f^{\prime}\right)-\Delta_{j}\left(n, x_{n}, z f_{n}^{\prime}\right)\right) / n^{2 p-j}=o(1) .
$$

The first statement of Theorem 3, which involves $f$ and $f_{n}$ as opposed to $f^{\prime}$ and $f_{n}^{\prime}$, now follows by a simple induction argument from (8) and Lemma 2 of [8].

To prove the first statement of Theorem 4, we put $x=e^{i \beta}$ and expand $H_{q}(n, f), H_{q}\left(n, f_{n}\right)$ by the symmetric group as in $[8, \mathrm{p} .519]$. We find that

$$
H_{q}(n, f)-H_{q}\left(n, f_{n}\right)=\sum_{\mu}\left\{\prod_{m=1}^{q} \Delta_{\mu(m)}(f)-\prod_{m=1}^{q} \Delta_{\mu(m)}\left(f_{n}\right)\right\},
$$

where $\mu$ is a permutation with $0 \leq \mu(m) \leq 2 q-2, \Sigma_{1}^{q} \mu(m)=q^{2}-q$. Now $p>q-3 / 4$ implies $p>(2 \mu(m)+1) / 4$, so from Theorem 3 ,

$$
\Delta_{\mu(m)}(f)=\Delta_{\mu(m)}\left(f_{n}\right)+o(1) n^{2 p-\mu(m)-1} .
$$

It is easily verified that $\Delta_{\mu(m)}\left(f_{n}\right)=O(1) n^{2 p-\mu(m)-1}$, and so

$$
\prod_{m=1}^{q} \Delta_{\mu(m)}(f)=\prod_{m=1}^{q} \Delta_{\mu(m)}\left(f_{n}\right)+o(1) n^{2 p q-q^{2}} \text {. }
$$

Thus from (9) and (10) we see that

$$
H_{q}(n, f) / n^{2 p q-q^{2}}=H_{q}\left(n, f_{n}\right) / n^{2 p q-q^{2}}+o(1) .
$$


Simple computations show that

$$
H_{q}\left(n, f_{n}\right)=\alpha_{n}(f)^{q} \exp (-i(n+q-1) q \beta) H_{q}\left(n, f_{0}\right),
$$

where $f_{0}(z)=(1-z)^{-2 p}$. Since $\left|\alpha_{n}(f)\right| \rightarrow \alpha(f)$ as $n \rightarrow \infty$, the first statement of Theorem 4 follows immediately from (1) and (11).

In order to show that the restrictions $p>(2 j+1) / 4$ and $p>q-3 / 4$ of Theorems 3 and 4 , respectively, are best possible, let $\left\{b_{n}\right\}$ be any sequence of nonne gative numbers satisfying $b_{1}=0, \Sigma_{1}^{\infty} b_{n} \leq 1, \Sigma_{2}^{\infty} n b_{n}^{2} \leq p$. Set $\xi(z)$ $=\Sigma_{1}^{\infty} b_{n} z^{n}$ and $F(z)=2^{2 p} \pi(1-z)^{-2 p}+\xi(z)$. By making obvious modifications in the argument given by Hayman [4, Lemma 9], we see that for $p \geq 1 / 2$, $F \in S_{p^{*}}$ (For notational ease we neglect the normalization constant.) It is clear that $\alpha(F)=\lim _{r \rightarrow 1}(1-r)^{2 p}|F(r)|>0$, no matter what the sequence $\left\{b_{n}\right\}$.

To prove that the restriction $p>(2 j+1) / 4$ in Theorem 3 is best possible, choose $\epsilon>0$ very small, set $p=(2 j+1) / 4-\epsilon$, and choose a sequence $\left\{\delta_{n}\right\}$ such that $\lim _{n \rightarrow \infty} \delta_{n}=0, \lim _{\sup _{n \rightarrow \infty}} \delta_{n} n^{2 \epsilon}=+\infty$. Now choose $\left\{b_{n}\right\}$ subject to the previous restrictions such that: (i) $b_{n}=\delta_{n} n^{-1 / 2}$ for infinitely many $n$; (ii) $\lim _{n \rightarrow \infty} \delta_{n} n^{2 \epsilon}=\infty$ for those integers chosen in (i); (iii) $b_{n}=0$ except for the integers chosen in (i); (iv) if $b_{n} \neq 0$, then $b_{n+1}=\cdots=b_{n+j}$ $=0$. (A specific example is as follows: choose an integer $c$ such that $c>$ $1 / \epsilon, m^{c}-(m-1)^{c}>j$ for all $m \geq 2$. If $k=m^{c}$ for some $m$, put $b_{k}=m^{-2 / k^{1 / 2}}$ $=m^{-2-c / 2}$, and put $b_{k}=0$ otherwise.)

Now let $\left\{e^{i \theta} n\right\}$ be given. If for some subsequence $\left\{n_{k}\right\}$ we have that $\lim _{k \rightarrow \infty} \exp \left(i \theta_{n_{k}}\right)=a \neq 1$, then as in the proof of [8, Theorem 4] we have that $\lim \sup _{n \rightarrow \infty} \Delta_{j}\left(n_{9} e^{i \theta_{n}}, G\right) / n^{2 p-j-1}=\infty$, where $G(z)=2^{2 p} \pi(1-z)^{-2 p}$. By using this fact in conjunction with the fact that

$$
\Delta_{j}\left(n, e^{i \theta_{n}}, F\right)=\Delta_{j}\left(n, e^{i \theta_{n}}, G\right)+\left(-e^{i \theta_{n}}\right)^{k}\left(\begin{array}{l}
j \\
k
\end{array}\right) b_{n+k}
$$

for some $k, 0 \leq k \leq j$ (this holds because of (iv) above), we conclude that $\lim \sup _{n \rightarrow \infty} \Delta_{j}\left(n, e^{i \theta} n, F\right) / n^{2 p-j-1}=\infty$, as required. If $\lim _{n \rightarrow \infty} e^{i \theta_{n}}=1$, choose a subsequence (which we denote by $\left\{e^{i \theta} n\right\}$ ) such that

$$
\psi=\lim _{n \rightarrow \infty} n\left(1-e^{i \theta_{n}}\right)
$$

exists and such that

$$
\limsup _{n \rightarrow \infty} \frac{\left|\Delta_{j}\left(n, e^{i \theta_{n}}, G\right)\right|}{n^{2 p-j-1}}=\lim _{n \rightarrow \infty} \frac{\mid\rfloor_{j}\left(n, e^{i \theta} n, G\right) \mid}{n^{2 p-j-1}}
$$


where $G(z)=2^{2 p} \pi(1-z)^{-2 p}$, and where it is understood that the lim sup in (12) is taken over the full sequence, while the limit is taken over the subsequence. Since $e^{i \theta_{n}} \rightarrow 1, \psi$ is purely imaginary, with $\pm i \infty$ as possible values. If $\psi= \pm i \infty$, equations (6.6) and (6.7) of [8] imply that

$$
\limsup _{n \rightarrow \infty}\left|\Delta_{j}\left(n, e^{i \theta} n, F\right)\right| / n^{2 p-j-1}=\infty \text {. }
$$

If $\psi$ is finite, it follows from (6.6) of [8] that the quantity in (12) is finite. We now choose another subsequence (again denoted by $\left\{e^{i \theta_{n}}\right\}$ ) such that $b_{n}=\delta_{n} n^{-1 / 2}, \delta_{n} n^{2 \epsilon} \rightarrow \infty$. Condition (iv) above guarantees that $\Delta_{j}\left(n, e^{i \theta_{n}}, F\right)$ $=\Delta_{j}\left(n, e^{i \theta_{n}}, G\right)+b_{n}$, and since $b_{n} n^{-2 p+j+1}=\delta_{n} n^{2 \epsilon}$, we deduce that $\lim \sup _{n \rightarrow \infty} \Delta_{j}\left(n, e^{i \dot{\theta}_{n}}, F\right) / n^{2 p-j-1^{n}}=\infty$ as required.

The proof that the restriction $p>q-3 / 4$ is best possible is very similar. With $F$ as before, set $p=q-3 / 4-6$. Choose $\left\{\delta_{n}\right\}$ such that $\lim _{n \rightarrow \infty} \delta_{n}=0$, $\lim _{m \rightarrow \infty} n_{m}^{2 \epsilon} \delta_{n_{m}}=+\infty$ for some subsequence $\left\{n_{m}^{n}\right\}$. If $k=n_{m}$ for some $m$, set $b_{k+2 q-2}=\delta_{k} k^{-1 / 2}$. Otherwise set $b_{k}=0$. Then with $G(z)^{m}=2^{2 p} \pi(1-z)^{-2 p}$, we have that

$$
H_{q}\left(n_{m}, F\right)=H_{q}\left(n_{m}, G\right)+(-1)^{q+1} H_{q-1}\left(n_{m}, G\right) b_{n_{m}}+2 q-2 .
$$

Upon combining this expression with (1), it follows that

$$
\limsup _{m \rightarrow \infty} \frac{H_{q}\left(n_{m}, F\right)}{n_{m}^{2 p q-q^{2}}}=\infty \quad \text { iff } \limsup _{m \rightarrow \infty} \frac{b_{m}+2 q-2}{n_{m}^{\tau}}=\infty,
$$

where $\tau=2 p+1-2 q$. But $2 p-2 q+1=-1 / 2-2 \epsilon$, and $b_{n_{m}+2 q-2}=\delta_{n_{m}} n_{m}^{-1 / 2}$, so

$$
\limsup _{m \rightarrow \infty} \frac{b_{n_{m}}+2 q-2}{n_{m}^{\tau}}=\lim _{m \rightarrow \infty} \sup _{m} \delta_{n_{m}} n_{m}^{2 \epsilon}=\infty \text {. }
$$

This completes the proof of Theorem 4.

5. Conclusion. If $f \in S_{p}$, then there exists an annulus $A(\delta, f)=$ $\{z: 1-2 \delta<|z|<1\}$ in which $f$ does not vanish, and so [5, p. 103] for any $\lambda>0, g(z)=f(z)^{\lambda}$ may be continued analytically in $A(\delta, f)$. There exists $\mu$ such that in $A(\delta, f), q(z) / z^{\mu}=\Sigma_{-\infty}^{+\infty} a_{n} z^{n}$.

It appears to be an open question as to whether or not $g$ is areally mean $\lambda p$-valent in $A(\delta, f)$. In any case, the main results of this paper continue to hold for such a $g$, provided we replace $p$ by $p \lambda$ throughout For example, if $q \geq 1, \alpha(f)>0$, and $p \lambda>q-3 / 4$, then 


$$
\lim _{n \rightarrow \infty} \frac{\left|H_{q}(n, g)\right|}{n^{2 p \lambda} \lambda_{q}-q^{2}}=\left(\frac{\alpha(f)}{\Gamma(2 p \lambda)}\right)^{q}\left|Q_{q}(p \lambda)\right| .
$$

The proofs for arbitrary $\lambda>0$ are straightforward generalizations of the proofs for $\lambda=1$, although the details become somewhat more complicated. These proofs are left to the interested reader.

\section{REFERENCES}

1. B. G. Eke, The asymptotic behaviour of areally mean valent functions, J. Analyse Math. 20 (1967), 147-212. MR 36 \#5331.

2. W. K. Hayman, The asymptotic behavior of p-valent functions, Proc. London Math. Soc. (3) 5 (1955), 257-284. MR 17, 142.

3. - On successive coefficients of univalent functions, J. London Math. Soc. 38 (1963), 228-243. MR 26 \#6382.

4. - On the second Hankel determinant of mean univalent functions, Proc. London Math. Soc. (3) 18 (1968), 77-94. MR 36 \#2794.

5. - Multivalent functions, Cambridge Tracts in Math. and Math. Phys., no. 48, Cambridge Univ. Press, Cambridge, 1958. MR 21 \#7302.

6. - Research problems in function theory, Athlone Press, London, 1967. MR 36 \#359.

7. K. W. Lucas, On successive coefficients of areally mean p-valent functions, J. London Math. Soc. 44 (1969), 631-642. MR 39 \#4379.

8. J. W. Noon an and D. K. Thomas, On the Hankel determinants of areally mean p-valent functions, Proc. London Math. Soc. (3) 25 (1972), 503-524. MR 46 \#605.

9. Ch. Pommerenke, On the coefficients and Hankel determinants of univalent functions, J. London Math. Soc. 41 (1966), 111-122. MR 32 \#2575.

10. - On the Hankel determinants of univalent functions, Mathematika 14 (1967), 108-112. MR 35\#6811.

DEPARTMENT OF MATHEMATICS, COLLEGE OF THE HOLY CROSS, WORCESTER, MASSACHUSE TTS 01610 